\title{
Mental Body Representations Retain Homuncular Shape Distortions: Evidence from Weber's Illusion
}

\author{
Luke E. Miller ${ }^{\mathrm{a}, \mathrm{b}}$ \\ Matthew R. Longo ${ }^{c}$ \\ Ayse P. Saygin ${ }^{\mathrm{a}, \mathrm{b}}$ \\ Affiliations \\ ${ }^{a}$ Department of Cognitive Science, University of California, San Diego \\ ${ }^{\mathrm{b}}$ Kavli Institute for Brain and Mind, University of California, San Diego \\ ${ }^{c}$ Department of Psychological Sciences, Birkbeck, University of London
}

Correspondence should be addressed to L.E. Miller

9500 Gilman Drive, Department of Cognitive Science,

University of California San Diego, La Jolla, CA, 92093-0515, USA.

E-mail: lumiller@ucsd.edu 


\begin{abstract}
Mental body representations underlying tactile perception do not accurately reflect the body's true morphology. For example, perceived tactile distance is dependent on both the body part being touched and the stimulus orientation, a phenomenon called Weber's illusion. These findings suggest the presence of size and shape distortions, respectively. However, whereas each morphological feature is typically measured in isolation, a complete morphological characterization requires the concurrent measurement of both size and shape. We did so in three experiments, manipulating both the stimulated body parts (hand; forearm) and stimulus orientation while requiring participants to make tactile distance judgments. We found that the forearm was significantly more distorted than the hand lengthwise but not widthwise. Effects of stimulus orientation are thought to reflect receptive field anisotropies in primary somatosensory cortex. The results of the present study therefore suggest that mental body representations retain homuncular shape distortions that characterize early stages of somatosensory processing.
\end{abstract}

Keywords: body representation; psychophysics; touch; perception; somatosensory 


\section{Introduction}

It has been known since the classic investigations of E.H. Weber in the $19^{\text {th }}$ century (Weber, 1834/1978) that the perceived distance between distinct points of touch is dependent upon the skin surface being touched. Two tactile points are perceived as farther apart on body parts with higher tactile sensitivity than those with lower sensitivity (e.g., fingers vs. thigh), a phenomenon known as Weber's illusion. Several studies with varying methods have since verified and expanded upon this classic finding (Anema, Wolswijk, Ruis, \& Dijkerman, 2008; Cholewiak, 1999; de Vignemont, Majid, Jola, \& Haggard, 2009; Goudge, 1918; Green, 1982; Le Cornu Knight, Longo, \& Bremner, 2014; Longo \& Haggard, 2011; Longo \& Sadibolova, 2013; Marks et al., 1982; Taylor-Clarke, Jacobsen, \& Haggard, 2004).

The neural mechanisms underlying Weber's illusion are at present poorly understood. Differences in tactile distance perception are often attributed to cortical magnification factors in primary somatosensory cortex (SI) (Cholewiak, 1999; Green, 1982). However, several recent studies have found that tactile distance perception is modulated by altered multisensory input (Miller, Longo, \& Saygin, 2014; TajaduraJiménez et al., 2012; Taylor-Clarke et al., 2004). Further, quantifications of the magnitude of Weber's illusion (Taylor-Clarke et al., 2004) found it to only be about 10\% of what would be expected from cortical magnification factors in SI (Penfield \& Boldrey, 1937). These findings suggest that Weber's illusion is also dependent upon later stages of somatosensory processing involved in the construction of higher-level mental body representations (Serino \& Haggard, 2010). These representations are likely located in the posterior parietal cortex (Bolognini \& Maravita, 2007; Duhamel, Colby, \& Goldberg, 1998) and arise from reciprocal interactions with SI (Cooke et al., 2014; Goldring et al., 2014).

Weber's illusion is commonly used to investigate the morphology of these higherlevel mental body representations. In one popular version of a tactile distance judgment task (TDJ), participants compare the perceived distance of two temporally distinct presentations of touch. Touch is typically administered to two distinct body parts, in order to quantify differences in the represented size of body parts (de Vignemont, 
Ehrsson, \& Haggard, 2005; Tajadura-Jiménez et al., 2012; Taylor-Clarke et al., 2004). It is inferred that the body part with the greatest perceived tactile distance is represented as larger than the other. Recently, the TDJ has been administered within the same body part, in order to investigate its represented shape (Longo \& Haggard, 2011). Participants made distance judgments about touch administered in two different orientations (transverse and longitudinal). Longo and Haggard found that tactile distance perception on the hand was greater in the transverse orientation, suggesting that the representation is short and squat. This finding converges with other methods for mapping perceived body shape (Longo \& Haggard, 2010), a continuity suggesting that Weber's illusion reflects distortions in body representation. However, this task is limited to measuring the aspect ratio within body parts and not shape differences between them. A full morphological characterization of mental body representations would require both size and shape to be measured concurrently. The present study takes the first steps towards this goal.

Here, we present a detailed investigation into the morphological properties of hand and forearm representations. We report three psychophysics experiments that investigated the effect of stimulus orientation on Weber's illusion between body parts. In all experiments, touch was presented in two orientations - transverse and longitudinalto each body part. In Experiment 1, tactile points presented to the hand were compared against tactile points presented to the arm. In Experiments 2 and 3, we isolated tactile distance perception on the hand and arm individually; tactile points presented to a target body part (Experiment 2: hand; Experiment 3: arm) were compared to a shared reference body part, the forehead, which is commonly used when measuring tactile distance perception on the hand and arm (e.g., Bassolino, Finisguerra, Canzoneri, Serino, \& Pozzo, 2015; Canzoneri et al., 2013; de Vignemont et al., 2005; Miller et al., 2014).

The results of these experiments have important implications for our understanding of the mechanisms underlying tactile distance perception generally, and Weber's illusion specifically. First, by manipulating orientation, we can concurrently measure differences in the represented size and shape of these body parts. This allows us to overcome the limitations of previous methods discussed earlier. Second, by measuring tactile distance perception on the arm and hand separately, we can investigate whether shape distortions measured in Experiment 1 are task-independent characteristics of 
mental body representations. Third, and most important, our finding would shed light on the neural mechanisms underlying Weber's illusion. Effects of stimulus orientation are thought to reflect anisotropies in receptive field (RF) geometry of SI neurons (Cody, Garside, Lloyd, \& Poliakoff, 2008; Fuchs \& Brown, 1984; Gibson \& Craig, 2005; Wheat \& Goodwin, 2000). Therefore, orientation-specific differences in Weber's illusion would suggest an intimate relationship between body representations at early and late stages of somatosensory processing (Serino \& Haggard, 2010).

Based on known differences in the size of the homuncular representations of the arm and hand (Penfield \& Boldrey, 1937), we hypothesized that we would find a significant Weber's illusion between the two body parts. We further hypothesized that the presence of a Weber's illusion between the arm and hand would reflect differences in RF shape. A model of Weber's illusion that links tactile distance perception to low-level properties of SI - the pixel model (Longo \& Haggard, 2011) - motivated these hypotheses.

\section{Methods}

\subsection{Participants}

Twenty right-handed adults (13 females) between 19 and 29 years of age (mean: 21.1; SD: 2.2) participated in Experiment 1; Twenty-five right-handed adults (14 females) between 19 and 34 (mean: 21.7; SD: 3.1) participated in Experiment 2; Twenty-five right-handed adults (20 females) between 18 and 34 (mean: 21,3; SD: 3.3) participated in

Experiment 3. Each participant took part in only one experiment. The institutional review board at the University of California, San Diego approved each experiment, and all participants gave written informed consent before participating.

\subsection{Tactile Distance Judgment Task}

The methods used in each experiment were almost identical. We measured Weber's illusion with the TDJ (Figure 1). On each trial, two pairs of tactile stimuli (two wooden posts, each tapered to a $1 \mathrm{~mm}$ flat point) were applied sequentially, one pair to a target body part and the other to a reference body part. Tactile stimuli were applied for approximately one second, with approximately two seconds in between the administration of each stimulus pair. The combination of body sites was the only factor 
that differed between experiments (target vs. reference); Experiment 1: hand dorsum vs. forearm dorsum; Experiment 2: hand dorsum vs. forehead; Experiment 3: forearm dorsum vs. forehead. Tactile stimuli were always delivered to the middle of the body part, and the dominant arm/hand (i.e., right). Participants sat blindfolded with their arms rested flat on a table and their fingers splayed. Their task on each trial was to judge whether the distance between the two posts felt farther apart on the target or on the reference site. The administered distances were 2, 3 or $4 \mathrm{~cm}$, applied in five distance combinations (target/reference: $2 / 4,2 / 3,3 / 3,3 / 2,4 / 2$ ).

Critically, we manipulated the orientation of stimuli across trials: on each trial, touch was presented either transversally (hand/arm: medio-lateral; forehead: eye-to-eye) or longitudinally (hand/arm: proximo-distal; forehead: naison-to-hairline). On each trial, stimulus orientation was always the same for target and reference body part. Each distance combination was applied eight times in each orientation for a total of 40 trials per orientation, and 80 trials in all. The body part stimulated first (target or reference) as well as stimulus orientation was counterbalanced across trials.

\subsection{Curve Fitting}

Logistic functions were fit to each participant's response profile for each orientation separately. The point of subjective equality (PSE) from each psychometric curve quantified the magnitude of Weber's illusion. The PSE, the point where the curve crosses the $50 \%$ mark on the $y$-axis, is a measure of the proportional difference between the distance applied to the target and reference body part required for the two stimuli to be perceived as equidistant. A PSE significantly greater than zero indicates that touch on the target body part felt farther apart than the reference; a PSE significantly less than zero indicates the opposite. Psychometric curves were fit with a maximum likelihood procedure (Wichmann \& Hill, 2001) using the Palamedes Toolbox (Prins, 2009) in MATLAB (The MathWorks, Natick, MA). Goodness of fit was assessed with $\mathrm{R}^{2}$ values. This procedure was identical for all three experiments.

The presence of a Weber's illusion for each experiment was assessed with a onesample $t$-test comparing the PSE against zero. Perceptual differences between stimulus orientations within each experiment were assessed using a paired $t$-test. We directly compared the results of Experiment 2 and Experiment 3 using a 2 x 2 Mixed Effects 
ANOVA with Orientation (longitudinal; transverse) as a within-subjects factor and Target (hand; arm) as a between-subjects factor.

\section{Results}

The results for all experiments can be seen in Table 1 and Figure 2.

\subsection{Experiment 1}

One participant was excluded from the analysis because their psychometric curve in one

orientation had a poor fit to the data $\left(R^{2}=0.50\right)$. The psychometric curves of the remaining nineteen participants had good fit to the data in both the transversal (mean: 0.97; range: 0.91-1) and longitudinal (mean: 0.95; range: 0.86-1) orientation.

We found a significant Weber's illusion for stimuli presented in the longitudinal axis of the hand and forearm, but not in the transverse axis (Figure 2a). Tactile points presented along the hand were perceived as $71.6 \%( \pm 8.18)$ farther apart than on the forearm when presented longitudinally $(t(18)=8.74, p<0.001, d=2.01)$. In contrast, no Weber's illusion was found when stimuli were presented transversally $(1.85 \% \pm 4.53$; $t(18)=0.41, p=0.69, d=0.09)$. Paired t-tests demonstrated that the perceptual asymmetry between the hand and forearm was greater in the longitudinal than in the transverse orientation $\left(t(18)=7.14, p<0.001, d_{z}=1.64\right)$.

\subsection{Experiment 2}

The psychometric curves of all participants had good fit to the data in both the transversal (mean: 0.98; range: 0.85-1) and longitudinal (mean: 0.96; range: 0.84-1) orientation.

Previous studies have shown that tactile spatial acuity is similar on the hand and forehead (e.g., Mancini et al., 2014), the target and reference sites of the present experiment. Several studies have also failed to find a Weber's illusion between the two body parts (de Vignemont et al., 2005; Taylor-Clarke et al., 2004). It is therefore not surprising that, in the current experiment, the evidence for a Weber's illusion in either orientation was weak (Figure 2b). The PSE extracted from each participant's psychometric function were not significantly greater than zero for touch presented longitudinally $(5.84 \% \pm 3.29 ; t(24)=1.91, p=0.068, d=0.38)$ or transversally $(6.74 \% \pm$ 
3.52; $t(24)=1.78, p=0.088, d=0.36)$. Further, the Weber's illusion between the hand and forehead, if it exists, was not different for each orientation $\left(t(24)=0.20, p=0.84, d_{z}=0.04\right)$.

\subsection{Experiment 3}

One participant was excluded from the analysis because their psychometric curve in one orientation had a poor fit to the data $\left(R^{2}=0.55\right)$. The psychometric curves of the remaining twenty-four participants had good fit to the data in both the transversal (mean: 0.96; range: 0.84-1) and longitudinal (mean: 0.91; range: 0.71-0.99) orientation.

We found a significant Weber's illusion in both orientations on the arm and forehead (Figure 2c). In the longitudinal orientation, equidistant tactile points were perceived as $16.09 \%( \pm 5.36)$ closer together on the arm than the forehead $(t(23)=-3.00$, $p=0.006, d=0.61)$, and $13.06 \%( \pm 2.13)$ farther apart on the arm than the forehead in the transverse orientation $(t(23)=6.11, p<0.001, d=1.25)$. The perceptual asymmetries between each orientation were significantly different $\left(t(24)=5.11, p<0.001, d_{z}=1.04\right)$.

\subsection{Experiment 2 (Hand-Forehead) vs. Experiment 3 (Arm-Forehead)}

To determine whether the findings of Experiment 1 generalize to instances where each body site was used as a target body part (with a common reference site), we compared the results of Experiment 2 and 3 using a 2 x 2 Mixed Effects ANOVA (See Methods; Figure $3)$. We found a significant main effect of stimulus orientation $(F(1,47)=17.20, p<0.001$,

$\left.\eta_{p}^{2}=0.27\right)$, consistent with our findings that orientation is an important factor in Weber's illusion. We also found a weak but significant main effect of target body part $\left(F(1,47)=4.08, p=0.049, \eta^{2}=0.08\right)$. Crucially, there was a significant main effect of orientation and target body $\operatorname{part}\left(F(1,47)=15.20, p<0.001, \eta_{p}^{2}=0.24\right)$ Post-hoc independent samples t-tests found a significant difference in the magnitude of the Weber's Illusion when touch was oriented longitudinally $(t(47)=3.52, p=0.001, d=1.01)$ but not transversally $(t(47)=1.50, p=0.14, d=0.43)$. These results mirror what we found in Experiment 1 .

\section{Discussion}

The present study investigated whether Weber's illusion between two body parts is dependent on stimulus orientation. Tactile distances on the hand and the arm were 
compared against each other (Experiment 1), or against a common reference body site, (Experiment 2: hand/forehead; Experiment 3: arm/forehead). A clear Weber's illusion was found in both Experiment 1 and 3. Crucially, we found strong evidence that when Weber's illusion was present, it was dependent upon stimulus orientation, which manifest itself in two distinct ways: First, the presence of Weber's illusion in the first place may depend on stimulus orientation (Experiment 1). Second, the direction of the Weber's illusion may depend on orientation (Experiment 3). Taken together, these results provide strong evidence that the size and shape of higher-level mental body representations is highly distorted.

\subsection{Effects of orientation as evidence for distorted body representation}

It is well established that tactile spatial acuity is dependent upon stimulus orientation (Cody et al., 2008; Fuchs \& Brown, 1984; Gibson \& Craig, 2005). The extent that this is also true for the perception of tactile distance, which is likely dependent upon late stages of somatosensory processing (Serino \& Haggard, 2010), has not received significant attention by researchers. Two recent studies using a within-body part TDJ have demonstrated effects of orientation on Weber's illusion for both the hand (Longo \& Haggard, 2011) and arm (Le Cornu Knight et al., 2014). Each body site showed the same systematic perceptual asymmetry, namely greater perceived tactile distance in the transverse axis.

The TDJ is often used as a method for investigating the spatial properties of mental body representations. The between-body part variant is typically used to assess differences in represented size (de Vignemont et al., 2005; Tajadura-Jiménez et al., 2012; Taylor-Clarke et al., 2004), whereas the within-body part variant has been used to assess a body parts represented shape by manipulating stimulus orientation (Longo \& Haggard, 2011). However, these methods alone do not allow for a complete characterization of the represented morphology of the body. To do so, we created a hybrid task of both TDJ methods, manipulating stimulus orientation between body parts. This allowed us to infer differences in both size and shape of the arm and hand.

When comparing the hand and arm in Experiment 1, we found a Weber's illusion in the longitudinal orientation only; more specifically, tactile distance was perceived as larger on the hand. What do these results say about the shape of mental body 
representations? A recent study using the within-body part TDJ (Le Cornu Knight et al., 2014) found that the shape of the forearm was more distorted than the hand. Namely, the shape of the arm is shorter and squatter than hand. In the context of this finding, our results suggest that the represented widths of the skin surface of the arm and hand are equivalent, whereas the represented length of the arm's skin surface is significantly more compressed compared to the hand. A schematic of this deduction can be seen in Figure 4 .

\subsection{Weber's illusion is task-independent}

Weber's illusion has been measured with several methods, including verbal size estimation (Green, 1982; Longo \& Sadibolova, 2013), finger aperture estimation (Anema et al., 2008), and perceptually comparing touch on two body parts (e.g., present study; Longo \& Haggard, 2011; Taylor-Clarke et al., 2004). How comparable these methods are is unknown. Despite using different methods, the effects of orientation we found in Experiment 1 are consistent with Green (1982), who found that verbal estimates of tactile distance were more distorted in the longitudinal axis on the arm compared to the hand. This is true even though he measured tactile perception on the glabrous surface of the skin, which is less perceptually distorted than the hairy surface where we stimulated (Longo \& Haggard, 2011; Mancini et al., 2014). This convergence in results suggests that Weber's illusion taps into a body representation where both skin surfaces are at least partially unified (Longo \& Haggard, 2012).

The present convergence with the results of Green (1982) — who used verbal size estimation - suggests that Weber's illusion is independent of response-type and task. Additionally, the similarity between our findings and those of Le Cornu Knight and colleagues (2014) further suggests that (access to) the morphological properties of the body representations underlying Weber's illusion are largely task-independent. This would explain why differences between the represented morphology of the arm and hand measured in Experiment 1 generalized across reference sites (Exp. 2 and 3). Future research should study the task-independence of Weber's illusion more directly.

\subsection{Potential neural mechanisms of Weber's illusion}

Several mechanisms have been proposed for tactile spatial acuity, including peripheral innervation density (Van Boven \& Johnson, 1994), cortical magnification (Duncan \& 
Boynton, 2007; Sathian \& Zangaladze, 1996; Tegenthoff et al., 2005), and RF geometry in SI (Cody et al., 2008; Gibson \& Craig, 2005). However, the relationship between measures of tactile spatial acuity and measures of Weber's illusion is not clear. These mechanisms mentioned above likely play some role in Weber's illusion (Cholewiak, 1999; Green, 1982), however they are not the entire story.

Several studies have found that tactile distance perception is modulated by changes in sensory input (Tajadura-Jiménez et al., 2012; Taylor-Clarke et al., 2004) and the conscious feeling of body size (de Vignemont et al., 2005), as well as brief use of a tool (Canzoneri et al., 2013; Miller et al., 2014). For example, Taylor-Clarke and colleagues (2004) found that sustained visual magnification of a body part modulated the magnitude of Weber's illusion. Importantly, this had no effect on two-point discrimination thresholds, a measure that likely depends on the aforementioned low-level mechanisms (Fuchs \& Brown, 1984). Higher-level tactile judgments, such as distance, therefore depend upon late stages of somatosensory processing, likely in posterior parietal cortex (Bolognini \& Maravita, 2007; Duhamel et al., 1998), that construct multisensory body representations (Serino \& Haggard, 2010).

Further evidence for the role of body representations beyond SI (Longo, Azanon, \& Haggard, 2010) in tactile distance perception comes from the quantification of the magnitude of Weber's illusion. Perceptual asymmetries in tactile spatial acuity mirror known differences in cortical magnification (Gibson \& Craig, 2005), whereas previous quantifications of the magnitude of Weber's illusion found it to only be about $10 \%$ of what would be expected from cortical magnification alone (Taylor-Clarke et al., 2004). Our findings are relatively consistent with this quantification. Unlike gap detection thresholds, which are 300\% larger along the longitudinal axis of the forearm than the hand (Gibson \& Craig, 2005), we only found a 71\% difference in perceived tactile distance when they were compared directly in Experiment 1. These findings suggest that body-part-specific cortical magnification in SI is rescaled at later stages of somatosensory processing to a more veridical representation of body size, allowing for more accurate perceptual judgments. This rescaling, however, is incomplete (i.e., underconstancy of tactile distance perception), and thus the mental body representation underlying Weber's illusion retains differences in the size of SI representations. 
This rescaling process is also incomplete in another respect-it does not fully account for anisotropic RF geometry in SI. According to a recent model of tactile distance perception, the pixel model, late stages of somatosensory processing involved in tactile distance perception assume that the RFs in SI are circular (Longo \& Haggard, 2011). While neurophysiology studies have found somewhat circular RFs for the glaborous surface of body parts (e.g. DiCarlo \& Johnson, 2002), RFs for the hairy surface are oval-shaped with their long axis running longitudinally (e.g. Alloway, Rosenthal, \& Burton, 1989; Brown, Fuchs, \& Tapper, 1975). According to the pixel model, rescaling the shape of RFs from oval to circular leads to distortions in the shape of mental body representations underlying Weber's illusion, a prediction that has been borne out empirically (Le Cornu Knight et al., 2014; Longo \& Haggard, 2011).

The present study, particularly the results of Experiment 1, provides further evidence that mental body representations underlying Weber's illusion retain anisotropies of SI RF geometry. Neurophysiological studies have found that the shape of RFs coding for the hairy skin surface become progressively more ovalized the more distal the location on the forelimb (Alloway et al., 1989; Brown et al., 1975). For example, RF sizes in longitudinal axis of cat SI neurons representing the legs get progressively larger from paw to foreleg, leading to increasing anisotropy (Alloway et al., 1989) - this pattern of progressive ovalization is not present in the transverse axis. Given the findings of Alloway and colleagues, the pixel model would predict the existence of a Weber's illusion between the hand and arm in the longitudinal only —our results reflect this prediction. More work is needed, however, to further evaluate the pixel model of Weber's illusion. By comparing perceptual differences between body parts (i.e., upper arm, thigh, etc.) as well as body surfaces (i.e., glabrous versus hairy skin), this future work could better elucidate the mechanisms underlying Weber's illusion. However, taken at face value, our results suggests that the body representations underlying Weber's illusion retains distortions that characterize the size and shape of the somatosensory homunculus (Penfield \& Boldrey, 1937).

\section{Conclusion}


In three experiments, we found that Weber's illusion was dependent on stimulus orientation. These results demonstrate that the morphology of the mental body representations underlying the illusion is distorted, and that this distortion reflects known characteristics of the somatosensory homunculus - more specifically, SI receptive field geometry. The finding that higher-order mental body representations retain properties of a lower level of sensory organization suggests an intimate relationship between early and late stages of somatosensory processing. The somatosensory homunculus likely serves as the 'building blocks' that construct mental representations underlying bodily awareness.

Acknowledgments: This research was supported by the Kavli Institute for Brain and Mind, UCSD. APS was additionally supported by NSF (CAREER BCS1151805) and DARPA; MRL by a European Research Council Starting Grant (ERC-2013-StG336050). We would like to thank Paola Rossi, Nicola Geiß, and Andrew Cawley-Bennett for their help collecting data. We would further like to thank Lisa Quadt for her helpful comments on an earlier draft of the manuscript. 


\section{References}

Alloway, K. D., Rosenthal, P., \& Burton, H. (1989). Quantitative measurements of receptive field changes during antagonism of GABAergic transmission in primary somatosensory cortex of cats. Experimental Brain Research, 78(3), 514-532.

Anema, H. A., Wolswijk, V. W., Ruis, C., \& Dijkerman, H. C. (2008). Grasping Weber's illusion: the effect of receptor density differences on grasping and matching. Cogn Neuropsychol, 25(7-8), 951-967.

Bassolino, M., Finisguerra, A., Canzoneri, E., Serino, A., \& Pozzo, T. (2015). Dissociating effect of upper limb non-use and overuse on space and body representations. Neuropsychologia, 70, 385-392.

Bolognini, N., \& Maravita, A. (2007). Proprioceptive alignment of visual and somatosensory maps in the posterior parietal cortex. Current Biology, 17(21), 1890-1895.

Brown, P. B., Fuchs, J. L., \& Tapper, D. N. (1975). Parametric studies of dorsal horn neurons responding to tactile stimulation. Journal of Neurophysiology, 38(1), 19-25.

Canzoneri, E., Ubaldi, S., Rastelli, V., Finisguerra, A., Bassolino, M., \& Serino, A. (2013). Tool-use reshapes the boundaries of body and peripersonal space representations. Experimental Brain Research, 228(1), 25-42.

Cholewiak, R. W. (1999). The perception of tactile distance: influences of body site, space, and time. Perception, 28(7), 851-875.

Cody, F. W., Garside, R., Lloyd, D., \& Poliakoff, E. (2008). Tactile spatial acuity varies with site and axis in the human upper limb. Neurosci Lett, 433(2), 103-108.

Cooke, D. F., Goldring, A. B., Baldwin, M. K., Recanzone, G. H., Chen, A., Pan, T., et al. (2014). Reversible deactivation of higher-order posterior parietal areas. I. Alterations of receptive field characteristics in early stages of neocortical processing. J Neurophysiol, 112(10), 2529-2544.

de Vignemont, F., Ehrsson, H. H., \& Haggard, P. (2005). Bodily illusions modulate tactile perception. Current Biology, 15(14), 1286-1290.

de Vignemont, F., Majid, A., Jola, C., \& Haggard, P. (2009). Segmenting the body into parts: evidence from biases in tactile perception. The Quarterly Journal of Experimental Psychology, 62(3), 500-512.

DiCarlo, J. J., \& Johnson, K. O. (2002). Receptive field structure in cortical area $3 \mathrm{~b}$ of the alert monkey. Behav Brain Res, 135(1-2), 167-178. 
Duhamel, J. R., Colby, C. L., \& Goldberg, M. E. (1998). Ventral intraparietal area of the macaque: congruent visual and somatic response properties. Journal of Neurophysiology, 79(1), 126-136.

Duncan, R. O., \& Boynton, G. M. (2007). Tactile hyperacuity thresholds correlate with finger maps in primary somatosensory cortex (S1). Cerebral Cortex, 17(12), 2878-2891.

Fuchs, J. L., \& Brown, P. B. (1984). Two-point discriminability: relation to properties of the somatosensory system. Somatosens Res, 2(2), 163-169.

Gibson, G. O., \& Craig, J. C. (2005). Tactile spatial sensitivity and anisotropy. Percept Psychophys, 67(6), 1061-1079.

Goldring, A. B., Cooke, D. F., Baldwin, M. K., Recanzone, G. H., Gordon, A. G., Pan, T., et al. (2014). Reversible deactivation of higher-order posterior parietal areas. II. Alterations in response properties of neurons in areas 1 and 2. J Neurophysiol, 112(10), 2545-2560.

Goudge, M. E. (1918). A qualitative and quantitative study of Weber's illusion. The American Journal of Psychology, 81-119.

Green, B. G. (1982). The perception of distance and location for dual tactile pressures. Perception \& Psychophysics, 31(4), 315-323.

Le Cornu Knight, C., Longo, M. R., \& Bremner, A. J. (2014). Categorical perception of tactile distance. Cognition, 131(2), 254-262.

Longo, M. R., Azanon, E., \& Haggard, P. (2010). More than skin deep: Body representation beyond primary somatosensory cortex. Neuropsychologia, 48(3), 655-668.

Longo, M. R., \& Haggard, P. (2010). An implicit body representation underlying human position sense. Proc Natl Acad Sci US A, 107(26), 11727-11732.

Longo, M. R., \& Haggard, P. (2011). Weber's illusion and body shape: anisotropy of tactile size perception on the hand. J Exp Psychol Hum Percept Perform, 37(3), 720-726.

Longo, M. R., \& Haggard, P. (2012). A 2.5-D representation of the human hand. $J$ Exp Psychol Hum Percept Perform.

Longo, M. R., \& Sadibolova, R. (2013). Seeing the body distorts tactile size perception. Cognition, 126(3), 475-481.

Mancini, F., Bauleo, A., Cole, J., Lui, F., Porro, C. A., Haggard, P., et al. (2014). Wholebody mapping of spatial acuity for pain and touch. Ann Neurol, 75(6), 917-924.

Marks, L. E., Girvin, J. P., Quest, D. O., Antunes, J. L., Ning, P., O’Keefe, M. D., et al. (1982). Electrocutaneous stimulation II. The estimation of distance between two points. Perception \& psychophysics, 32(6), 529-536. 
Miller, L. E., Longo, M. R., \& Saygin, A. P. (2014). Tool morphology constrains the effects of tools on body representations. Journal of Experimental Psychology-Human Perception and Performance, 40(6), 2143-2153.

Penfield, W., \& Boldrey, E. (1937). Somatic motor and sensory representation in the cerebral cortex of man as studied by electrical stimulation. Brain, 60(4), 389-443.

Prins, N. (2009). Palamedes: Matlab routines for analyzing psychophysical data.

Sathian, K., \& Zangaladze, A. (1996). Tactile spatial acuity at the human fingertip and lip Bilateral symmetry and interdigit variability. Neurology, 46(5), 1464-1464.

Serino, A., \& Haggard, P. (2010). Touch and the body. Neurosci Biobehav Rev, 34(2), 224-236.

Tajadura-Jiménez, A., Väljamäe, A., Toshima, I., Kimura, T., Tsakiris, M., \& Kitagawa, N. (2012). Action sounds recalibrate perceived tactile distance. Curr Biol, 22(13), R516517.

Taylor-Clarke, M., Jacobsen, P., \& Haggard, P. (2004). Keeping the world a constant size: object constancy in human touch. Nat Neurosci, 7(3), 219-220.

Tegenthoff, M., Ragert, P., Pleger, B., Schwenkreis, P., Förster, A.-F., Nicolas, V., et al. (2005). Improvement of tactile discrimination performance and enlargement of cortical somatosensory maps after $5 \mathrm{~Hz}$ rTMS. PLoS biology, 3(11), e362.

Van Boven, R. W., \& Johnson, K. O. (1994). A Psychophysical Study of the Mechanisms of Sensory Recovery Following Nerve Injury in Humans. Brain, 117, 149-167.

Weber, E. (1834/1978). De subtilitate tactus. HE Ross \& DH Murray (HE Ross, Trans.). EH Weber: The sense of touch. London: Academic Press.(Originally published 1834).

Wheat, H. E., \& Goodwin, A. W. (2000). Tactile discrimination of gaps by slowly adapting afferents: effects of population parameters and anisotropy in the fingerpad. Journal of Neurophysiology, 84(3), 1430-1444.

Wichmann, F. A., \& Hill, N. J. (2001). The psychometric function: I. Fitting, sampling, and goodness of fit. Percept Psychophys, 63(8), 1293-1313. 


\begin{tabular}{lcccc}
\hline & Target & Reference & Transverse & Longitudinal \\
\hline Experiment 1 & Hand & Arm & 2.00 & $71.07^{* * *}$ \\
Experiment 2 & Hand & Head & $6.74^{\wedge}$ & $5.84^{\wedge}$ \\
Experiment 3 & \multirow{2}{*}{ Arm } & Head & $12.95^{* * *}$ & $-15.78^{* *}$ \\
\hline
\end{tabular}

\section{Table 1.}

Percent difference in tactile distance perception on the target and reference body parts. Positive numbers indicate a perceptual asymmetry in favor of touch on the target body part. Negative numbers indicate the opposite. Numbers significantly different than zero indicate the presence of a Weber's illusion on that body part for stimuli presented in that orientation.

$\wedge 0.05<p<0.1, * p<0.05, * * p<0.01, * * * p<0.001$ 


\section{Figure Captions}

Figure 1: Tactile Distance Judgment Task

The present figure depicts the procedures for Experiment 1. Participants are touched on the hand (dashed outline) and after a variable amount of time, the arm (solid outline). They then make an unspeeded judgment about the distance between the two points of touch (black dots), in this case saying that that they perceived the touch on the hand as farther apart than on the arm.

Figure 2: Results of all Experiments

The present figure depicts the group-level psychophysical curves (left panel) and bar plots of the PSEs (right panel) for each orientation (longitudinal: green; transverse: blue) and for each experiment (a: Experiment 1; b: Experiment 2; c: Experiment 3). The dashed lines indicate the point of subjective equality (PSE) for each curve, which can be seen in bar plot form in the right panels. A significant difference between the PSEs for each orientation indicates that the represented shape of the target body part is distorted. Error bars depict one standard error of the mean.

n.s. $=$ not significant; $* * *=p<0.001$

Figure 3: Comparison of Hand and Arm PSEs

The present figure shows PSE differences between hand (dashed colors) and arm (solid colors) for each orientation (longitudinal: green; transverse: blue). These results are consistent with Experiment 1, where the hand and arm were compared directly, suggesting that Weber's illusion may be task-independent. Error bars depict one standard error of the mean.

$$
\text { n.s. }=\text { not significant; } * * *=p<0.001
$$


Figure 4: Schematic of the arm and hand representations

The two arms on the left depict a participant's physical body. The colored dots represent the two tactile points applied in the transverse (blue) and longitudinal (green) orientations. The bidirectional arrows depict the perceived distance between the two tactile points for the arm (A) and hand $(\mathrm{H})$. As can be seen, the perceived distance only differs for touch administered in the longitudinal orientation. These results are then used to deduce the represented morphology of the arm and hand (animation on the right). In keeping with known shape distortions in the body representation underlying Weber's illusion (Le Cornu Knight et al., 2014; Longo \& Haggard, 2011), the hand and arm are depicted as being short and squat. However, the length of the arm is significantly more compressed compared to the hand, as can be seen in our depiction of the body representation. 


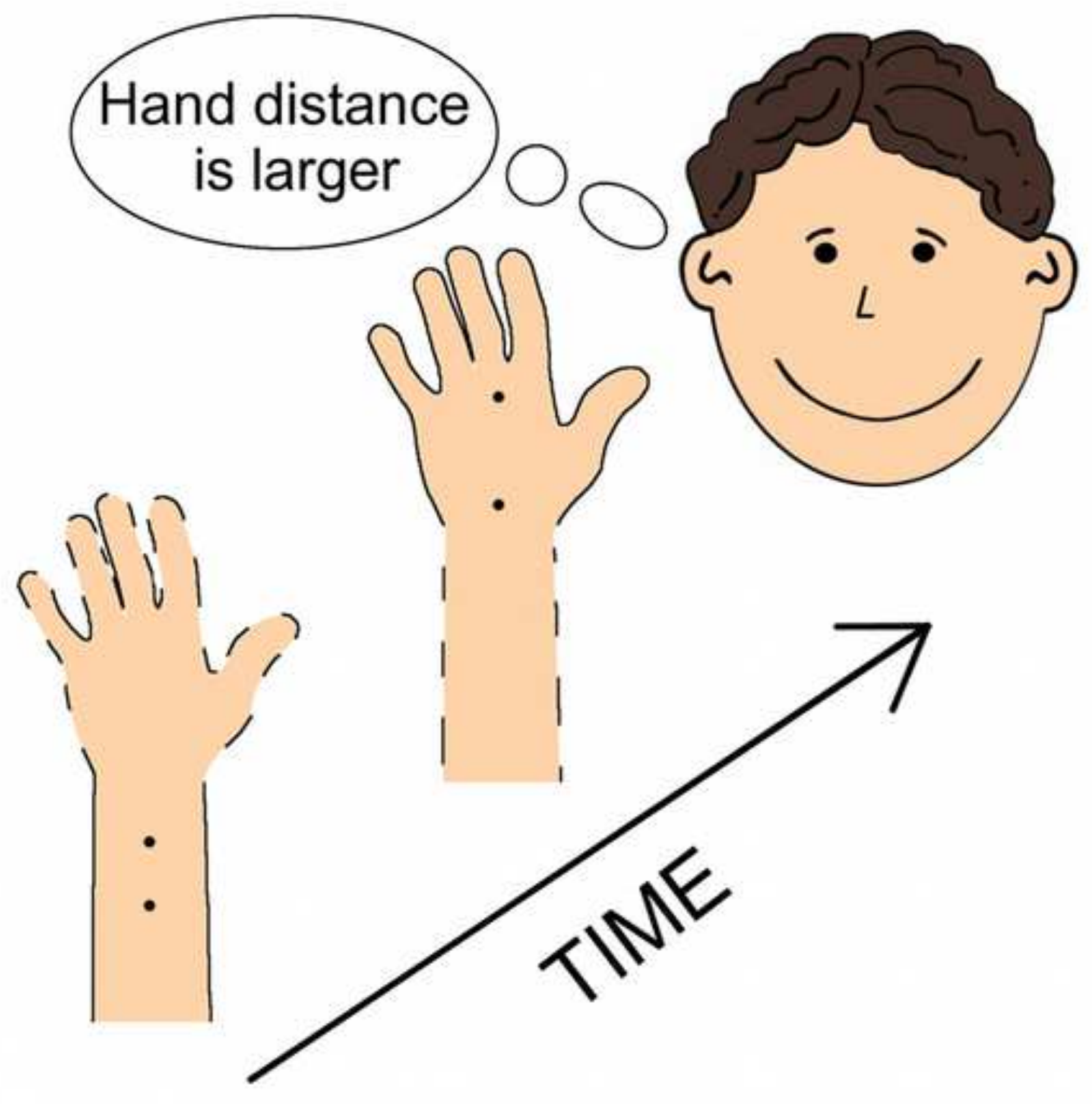


Exp. 1

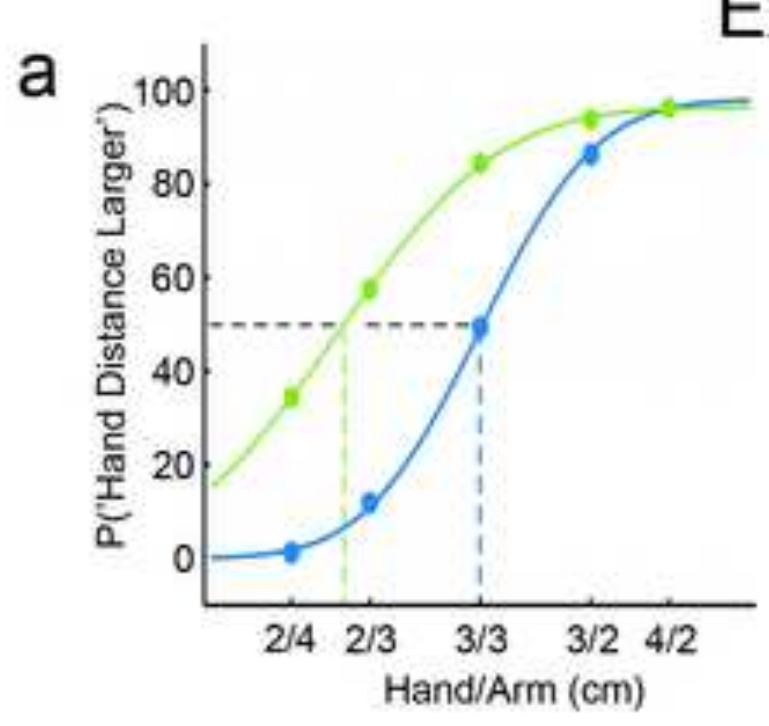

Exp. 2 b

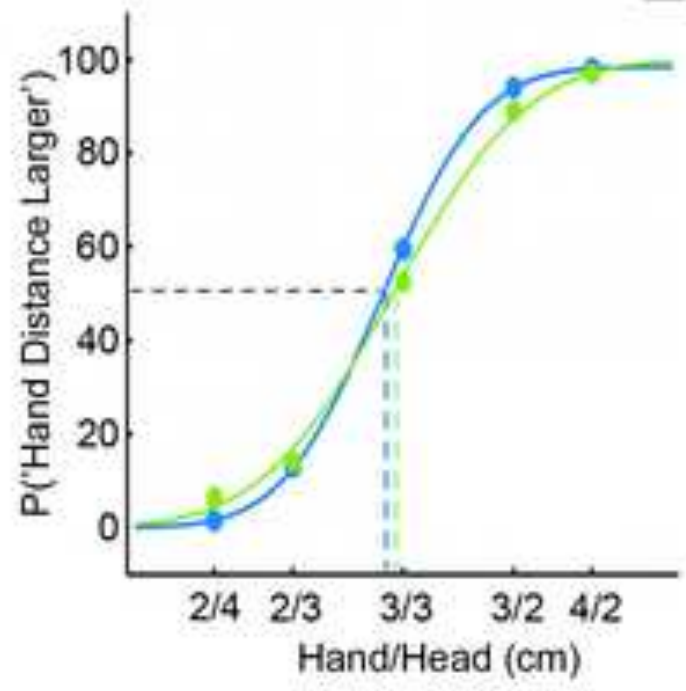

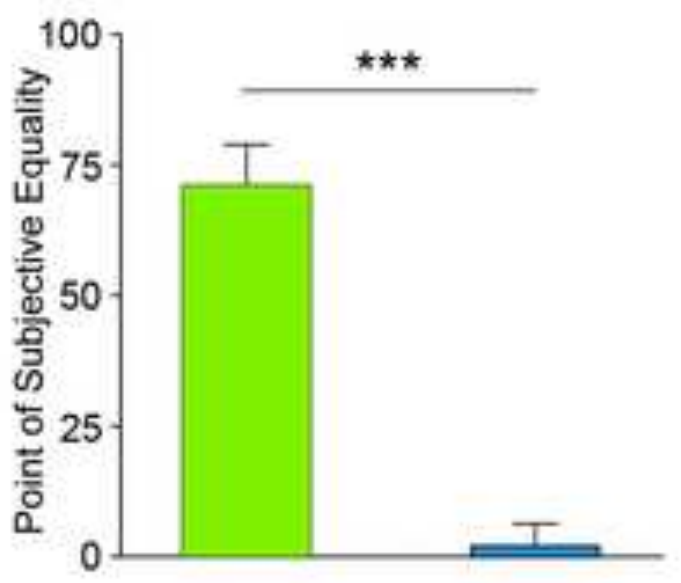

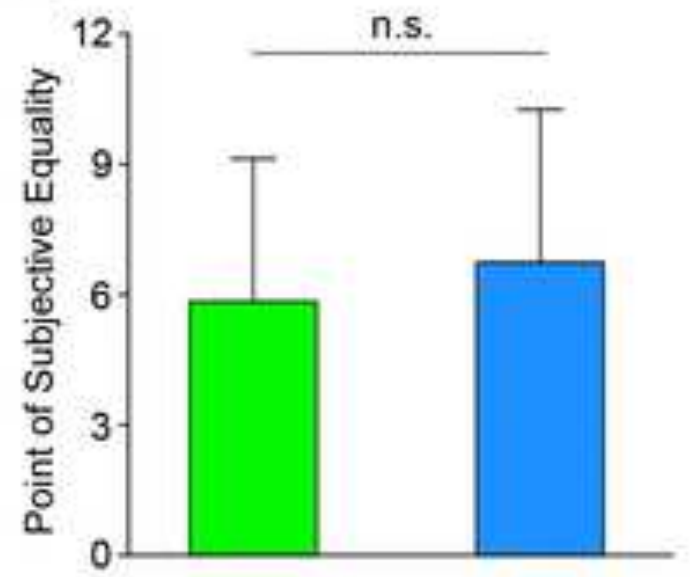

Exp. 3
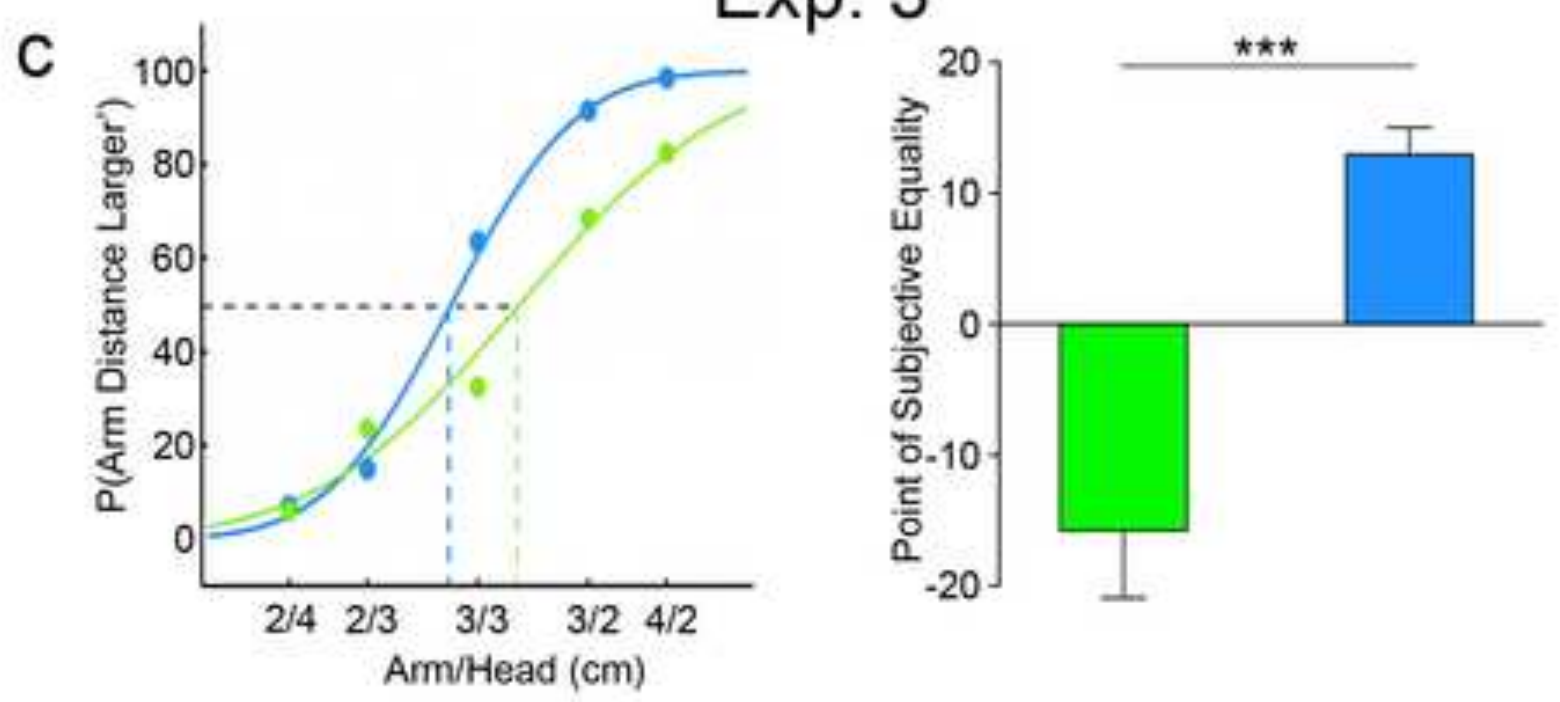


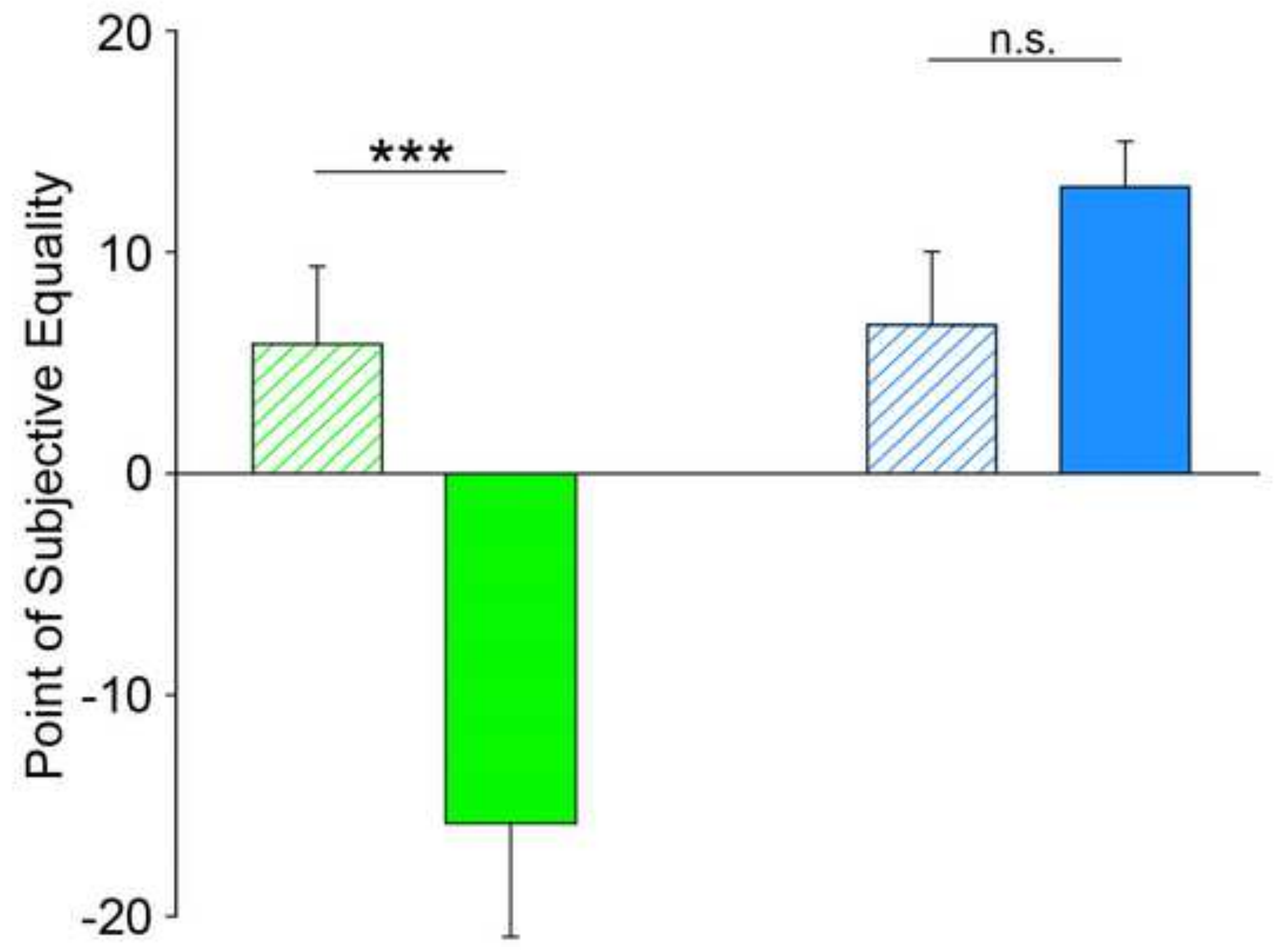

\section{Longitudinal}

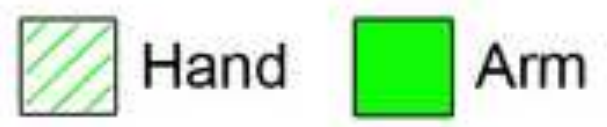

\section{Transverse}

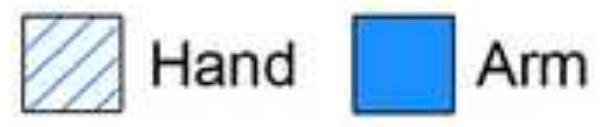


